\title{
Overnight in-hospital observation following tonsillectomy: retrospective study of post-operative intervention
}

\author{
S MORRIS $^{1}$, E HASSIN $^{2}$, M BORSCHMANN $^{2}$ \\ ${ }^{1}$ Princess of Wales Hospital, Bridgend, Wales, UK, and ${ }^{2}$ Department of ENT, University Hospital Geelong, \\ Victoria, Australia
}

\begin{abstract}
Objective: The safety of day-case tonsillectomy is widely documented in the literature; however, there are no evidence-based guidelines recommending patient characteristics that are incompatible with day-case tonsillectomy. This study aimed to identify which patients should be considered unsafe for day-case tonsillectomy based on the likelihood of needing critical intervention.

Method: Retrospective review of 2863 tonsillectomy procedures performed at University Hospital Geelong from 1998 to 2014.

Results: Of the patients, 7.81 per cent suffered a post-tonsillectomy complication and 4.15 per cent required intervention. The most serious complications, haemorrhage requiring a return to the operating theatre and airway compromise, occurred in 0.56 per cent and 0.11 per cent of patients respectively. The following patient characteristics were significantly associated with poorer outcomes: age of two years or less $(p<0.01)$, tonsillectomy indicated for neoplasm $(p<0.01)$ and quinsy $(p<0.05)$.

Conclusion: The authors believe that all elective tonsillectomy patients should be considered for day-case surgery, with the following criteria necessitating overnight observation: age of two years or less; an indication for tonsillectomy of neoplasm or quinsy; and an American Society of Anesthesia score of more than 2.
\end{abstract}

Key words: Tonsillectomy; Postoperative Hemorrhage; Day Surgery; Intraoperative Complications; Postoperative Complications

\section{Introduction}

Tonsillectomy represents $20-40$ per cent of the total procedures performed in ENT departments. ${ }^{1}$ Indications for tonsillectomy include recurrent tonsillitis, quinsy, tonsil neoplasm and obstructive sleep apnoea. ${ }^{1}$ Between 2012 and 2013, the overall rate of adenotonsillectomy in Australian public hospitals was approximately 4 per 1000. This equates to 52297 tonsillectomies in total. ${ }^{2}$

The safety of day-case tonsillectomy has been well documented in the literature. ${ }^{3-5}$ However, there are a number of serious complications which may make an overnight stay safer, including severe post-tonsillectomy haemorrhage and airway compromise. Airway complications including desaturation and stridor have been estimated to occur in up to 11.2 per cent of cases; some of these patients may even require re-intubation. The overall rates of post-tonsillectomy haemorrhage are typically $3-5$ per cent, with less than 1 per cent requiring a return to the operating theatre. ${ }^{6}$ Most post-tonsillectomy haemorrhage occurs at days 5-7 post-tonsillectomy, when patients have already been discharged. ${ }^{7-10}$ Identifying patients at highest risk for serious complications during the first 24 hours postoperatively would allow them to be excluded from day-case surgery.

Minor post-operative complications are estimated to occur in up to 20 per cent of cases; however, patients with these complications are considered safe for discharge, with appropriate management at home. ${ }^{11,12}$ These complications include pain, nausea, vomiting and fever, which may be managed with analgesics, anti-emetics or antibiotics. ${ }^{11-13}$

Literature suggests that a number of factors increase the risk of post-operative complications requiring intervention. These include: patient factors, such as age of two years or less, and a high American Society of Anesthesia Physical Status Classification score because of major heart disease, bleeding diatheses or airway 
disorders; and surgeon factors, such as diathermy use during the procedure. ${ }^{8-12,14,15}$

Multiple studies advocating day-case tonsillectomy have proposed selection criteria based on individual institutional experiences and the lack of complications occurring as a result of their selection processes. However, there are no evidence-based guidelines recommending which patients are safe for day-case tonsillectomy. ${ }^{2-6}$

This study primarily aimed to determine which patients required significant intervention during the first 24 hours following tonsillectomy or adenotonsillectomy performed at a single institution over a 16-year period. The study also aimed to determine how the findings may apply to future evidence-based guidelines for safe patient selection for day-case tonsillectomy.

\section{Materials and methods}

\section{Ethical considerations}

The Barwon Health - University Hospital Geelong granted permission to access and review patient data from June 1998 to June 2014.

\section{Participants and data sources}

A long-term retrospective review was conducted of 2863 tonsillectomy and adenotonsillectomy procedures performed at the University Hospital Geelong from June 1998 to June 2014. Data were generated by reviewing electronic and hard copy patient records, including progress notes, observation charts, medication charts, operation reports and discharge forms from the patient's stay in hospital following tonsillectomy.

Inclusion criteria. Patients of all ages undergoing tonsillectomy or adenotonsillectomy were included.

Exclusion criteria. Patients undergoing adenoidectomy alone or tonsillectomy as part of a wider procedure (e.g. neck dissection) were excluded.

\section{Study design}

Data collected from the above sources included: patient characteristics, such as age and gender; admission and re-admission details, such as admission, operation and discharge dates, length of stay, post-operative presentation to emergency department, and a return to the operating theatre; operative details, such as operative indication, American Society of Anesthesia score (1-4) and operative technique; and post-operative details, such as post-operative destination, observation time, nursing interventions (oxygen saturations, supplemental oxygen, analgesic and anti-emetic agents) and medical officer intervention (for pain, nausea, bleeding, sedation, fever and airway compromise).

All patients requiring intervention of any kind during their overnight admission were classified into four groups - mandatory, desirable, convenient and trivial stays - according to the reason for intervention.
Mandatory stay represented instances where an overnight observation was strongly indicated and sameday discharge would have been hazardous. This included cases of significant airway compromise or severe primary bleeds likely to require a return to the operating theatre. Desirable stay reflected instances where overnight observation was helpful, but sameday discharge was also safe. It included cases of sedation, fever, or lesser primary tonsil or adenoid bleeding managed on the ward. Convenient stay represented instances where overnight observation was not necessarily indicated but was convenient; and sameday discharge would have been safe. It included cases requiring an analgesic or anti-emetic related upgrade. Trivial stay concerned instances where a patient had miscellaneous problems, including unrelated symptoms or social issues.

This classification allows patient categorisation and observations regarding any shared characteristics of patients within the four groups, thus indicating favourable or unfavourable patient characteristics that would not be suited to day-case tonsillectomy.

\section{Statistical analysis}

EpiData software was used to collect and analyse the data. Categorical variables are presented as numbers and percentages, and continuous variables as means (ranges), unless otherwise stated. Further analysis involved chi-square testing, generating $p$-values, relative risk values and odds ratios.

\section{Results}

A total of 3098 cases were identified from the time period, of which 2863 cases met the inclusion criteria and comprised the study population.

The mean age of the sample was 20.71 years (range, 11 months to 79 years). Of the sample, 54.9 per cent were male, while 45.15 were female. Twenty-four of the patients were aged two years or less at the time of their operation ( 0.84 per cent) (Table I).

In this study, conducted at the University Hospital Geelong, the majority of tonsillectomy procedures were indicated by recurrent tonsillitis $(n=1840$, 64.27 per cent). Patients with sleep-disordered breathing represented 30.21 per cent of cases $(n=865)$ and those with quinsy represented 4.51 per cent $(n=$ 129); neoplasm was the least common indication for tonsillectomy ( $n=29,1.01$ per cent).

Of the cases included in this study, 1750 (61.12 per cent) were tonsillectomy and 1113 (38.88 per cent) were adenotonsillectomy. The majority of procedures (78.31 per cent) were performed using the 'hot' method in the form of bipolar or monopolar diathermy, against 21.69 per cent performed using the 'cold' steel method.

The University Hospital Geelong practice during the study period was to admit tonsillectomy patients for at least 1 night's observation. This was adhered to in 92.21 per cent of cases; however, 5.69 per cent were 


\begin{tabular}{lc}
\multicolumn{2}{c}{ TABLE I } \\
\multicolumn{2}{c}{ BACKGROUND CHARACTERISTICS } \\
\hline Variable & Patients $(n(\%))^{*}$ \\
\hline Age & $24(0.84)$ \\
$-\leq 2$ years & $2839(99.16)$ \\
$->2$ years & $20.71(11 \mathrm{mth}-79 \mathrm{y})$ \\
- Mean (range) & $1573(54.94)$ \\
Male gender & $1840(64.27)$ \\
Operative indication & $865(30.21)$ \\
- Recurrent tonsillitis & $129(4.51)$ \\
- Sleep-disordered breathing & $29(1.01)$ \\
- Quinsy & $1750(61.12)$ \\
- Tonsil neoplasm & $1113(38.88)$ \\
Operation performed & $621(21.69)$ \\
- Tonsillectomy & $2079(78.31)$ \\
- Adenotonsillectomy & $163(5.69)$ \\
Operative technique & - Cold steel \\
- Diathermy & $60(2.10)$ \\
Length of primary admission & - Same-day discharge \\
- Overnight & - >1 night
\end{tabular}

Total $n=2863$. *Unless units indicated as being otherwise. Mth $=$ months; $y=$ years

discharged the same day. Of the cases, 2.10 per cent were kept for a subsequent (more than 1) night. This means that a total of 2700 patients were kept for at least 1 night following their procedure and 163 were day-case admissions.

As part of their care, 96.72 per cent of patients (2769 out of 2863) were given an analgesic agent and 41.98 per cent $(n=1202)$ an anti-emetic post-operatively.

Of the 2700 patients who stayed for 1 night or more (2640 stayed 1 night and 60 stayed more than 1 night), 211 (7.81 per cent) had post-operative complications reported; 112 (4.15 per cent) required a clinical intervention for these complications from a medical officer.

The primary aim of this study was to determine what interventions were required during the in-patient admission; however, re-admission data have also been included. Thus, the rates of post-operative complications comprise those that occurred in both the primary (less than 24 hours post-operatively) and secondary (more than 24 hours post-operatively) periods.

Pain was the most common reason for intervention or review, occurring in 62 patients (2.30 per cent), of which 51 (1.89 per cent) required intervention in the form of an analgesic upgrade. Nausea occurred in 44 patients (1.63 per cent), with an intervention of antiemetic upgrade required in 12 patients $(0.44$ per cent). Primary bleeds occurred in 40 patients (1.48 per cent), of which 21 ( 0.78 per cent) were managed medically and 15 ( 0.56 per cent) required a return to the operating theatre. Fever was reported in 35 patients ( 1.30 per cent), and 2 patients ( 0.07 per cent) required intervention with antibiotics. Sedation was reported in 12 patients $(0.44$ per cent), and 2 patients $(0.07$ per cent) required intervention. All three patients with

\begin{tabular}{|c|c|c|}
\hline POST-OPERATIVI & $\begin{array}{c}\text { TABLE II } \\
\text { COMPLICATION } \\
\text { PATIENTS }\end{array}$ & N NON-DAY-CASE \\
\hline $\begin{array}{l}\text { Post-operative } \\
\text { complaint }\end{array}$ & $\begin{array}{l}\text { Complications } \\
\text { reported }(n(\%))\end{array}$ & $\begin{array}{c}\text { Medical officer } \\
\text { clinical intervention } \\
(n(\%))\end{array}$ \\
\hline Pain & $62(2.30)$ & $51(1.89)$ \\
\hline Nausea & $44(1.63)$ & $12(0.44)$ \\
\hline Primary bleed & $40(1.48)$ & \\
\hline $\begin{array}{l}\text { - Medical } \\
\text { management }\end{array}$ & & $21(0.78)$ \\
\hline $\begin{array}{l}\text { - Surgical return to } \\
\text { operating theatre }\end{array}$ & & $15(0.56)$ \\
\hline Fever & $35(1.30)$ & $2(0.07)$ \\
\hline $\begin{array}{l}\text { Sedation or GCS } \\
\text { score }<15\end{array}$ & $12(0.44)$ & $2(0.07)$ \\
\hline $\begin{array}{l}\text { Airway } \\
\text { compromise }\end{array}$ & $3(0.11)$ & $3(0.11)$ \\
\hline $\begin{array}{l}\text { Other or trivial } \\
\text { complaint }\end{array}$ & $15(0.56)$ & $6(0.21)$ \\
\hline Total patients & $211(7.81)$ & $112(4.15)$ \\
\hline
\end{tabular}

Total $n=2700$. Number of patients with no complaint reported $=$ 2489 (92.19 per cent). GCS = Glasgow Coma Scale

airway compromise required intervention ( 0.11 per cent). The 15 cases $(0.56$ per cent) of other complications included trivial complaints and unrelated symptoms, of which 6 ( 0.21 per cent) required intervention.

As reported in Table II, 15 patients required a return to the operating theatre for their primary bleed and 3 had airway compromise following the procedure. Of the 15 who required a return to the operating theatre, 1 patient had Von Willebrand disease and 6 patients had an American Society of Anesthesia score of more than 2 (Table III). Three of the returns to the operating theatre were indicated for tonsillectomy because of neoplasm, three because of quinsy, two because of sleep-disordered breathing and seven because of

\begin{tabular}{|c|c|c|}
\hline $\begin{array}{r}\text { CHARACTERIS } \\
\text { HAV }\end{array}$ & $\begin{array}{l}\text { TABLE III } \\
\text { OF PATIENTS } \\
\text { MANDATORY S }\end{array}$ & *SIFIED AS \\
\hline Variable & $\begin{array}{l}\text { Mandatory stay } \\
\text { patients }(n(\%))^{\dagger}\end{array}$ & $\begin{array}{l}\text { All patients } \\
(n(\%))^{\ddagger}\end{array}$ \\
\hline Age & & \\
\hline$-\leq 2$ years & $3(16.67)$ & $3 / 24(12.50)$ \\
\hline$->2$ years & $15(83.33)$ & \\
\hline $\begin{array}{l}\text { Associated } \\
\text { co-morbidity }\end{array}$ & & \\
\hline $\begin{array}{l}\text { - Von Willebrand } \\
\text { disease }\end{array}$ & $1(5.56)$ & \\
\hline - ASA score $>2$ & $6(33.33)$ & \\
\hline Operative indication & & \\
\hline - Neoplasm & $3(16.67)$ & $3 / 29(10.34)$ \\
\hline - Quinsy & $3(16.67)$ & $3 / 129(2.33)$ \\
\hline $\begin{array}{l}\text { - Sleep-disordered } \\
\text { breathing }\end{array}$ & $5(27.78)$ & $2 / 865(0.23)$ \\
\hline - Tonsillitis & $7(38.89)$ & $7 / 1840(0.38)$ \\
\hline Operative technique & & \\
\hline - Cold steel & $6(33.33)$ & 6/621 (0.97) \\
\hline - Diathermy & $12(66.67)$ & $12 / 2079(0.58)$ \\
\hline
\end{tabular}

* Because of a return to the operating theatre for adenotonsillar haemorrhage or airway compromise. ${ }^{n} n=18 ;{ }^{\star} n=2863$. ASA $=$ American Society of Anesthesia 
tonsillitis. The cold-steel technique was used in 33.33 per cent of the return to the operating theatre cases $(n=6)$ and diathermy in 66.67 per cent $(n=9)$.

Airway compromise post-procedure was indicated by stridor post-operatively. Of the three patients who had airway compromise, all were aged less than two years old, and two had an American Society of Anesthesia score of 2 or more because of gastric reflux. All patients were admitted to the intensive care unit, of whom one required intubation and two were further observed on the high dependency unit before discharge. The airway complications described occurred between 4 and 10 hours post-tonsillectomy, after the patients had left the recovery room and once they were on the ward. In all instances, a medical emergence team call was made. In the first case, the airway complications were due to sedation secondary to anaesthetic agents, and in the second case they were due to post-operative opiate-induced sedation. The third case of airway compromise was due to post-operative loss of positive end-expiratory pressure, desaturation and hypercapnia following the removal of large tonsils and adenoids, causing stridor and tachypnoea; this patient was re-intubated.

To put the Table III findings into context, 12.50 per cent of all patients aged two years or less required a mandatory stay because of post-operative complications. In addition, 10.34 per cent of all neoplasm cases ( 3 out of 29), 2.33 per cent of all quinsy cases (3 out of 129), 0.23 per cent of all sleep-disordered breathing cases ( 2 out of 865 ) and 0.38 per cent of all tonsillitis cases ( 7 out of 1840) required a mandatory stay.

Subsequently, statistical analysis was performed on all characteristics found to be common in those suffering mandatory complications.

Being two years of age or less at time of surgery was significantly associated with complications that would suggest a day stay to be unsafe (mandatory stay, $p=0.0004$ ), and resulted in a far higher risk of airway compromise or serious haemorrhage (relative risk $=23.66$, odds ratio $=26.90$; Table IV).

With regard to indications for tonsillectomy, quinsy and tonsil neoplasm were both significantly associated with reaching mandatory stay criteria ( $p=0.03707$ and $p=0.00067$ respectively; Table IV), with the former increasing the chance by 4 times (relative risk = 4.239 , odds ratio $=4.316$ ) and the latter by nearly 20 times (relative risk $=19.55$, odds ratio $=21.685$ ). Tonsillitis as an indication for tonsillectomy was significantly associated with a safe outcome (relative risk $=0.35$, odds ratio $=0.351, p=0.01727$ ), and sleep-disordered breathing was insignificantly associated with a safer outcome (relative risk $=0.82$, odds ratio $=0.766, p=0.076642$ ).

In contrast to evidence in the literature, ${ }^{15}$ there were no significant findings to suggest that cold-steel dissection was superior to diathermy in this sample (relative risk $=1.674$, odds ratio $=1.68, p=0.11947$ ).

\begin{tabular}{lcccc}
\multicolumn{5}{c}{ TABLE IV } \\
\multicolumn{5}{c}{ STATISTICAL FINDINGS FOR PATIENT } \\
CHARACTERISTICS AND OUTCOMES* \\
\hline Variable & $\begin{array}{c}\text { Relative } \\
\text { risk }\end{array}$ & $\begin{array}{c}\text { Odds } \\
\text { ratio }\end{array}$ & $\begin{array}{c}\text { Pearson's } \\
\text { chi-square }\end{array}$ & $p$ \\
\hline $\begin{array}{l}\text { Age } \leq 2 \text { years } \\
\text { Operative } \\
\text { indication }\end{array}$ & 23.66 & 26.90 & 54.595 & 0.00038 \\
- Tonsillitis & 0.35 & 0.351 & 5.081 & 0.01727 \\
$-\begin{array}{l}\text { Sleep- } \\
\text { disordered } \\
\text { breathing }\end{array}$ & 0.82 & 0.766 & 3.135 & 0.076642 \\
$-\begin{array}{l}\text { Quinsy } \\
\text { - Neoplasm }\end{array}$ & 4.239 & 4.316 & 6.226 & 0.03707 \\
$\begin{array}{l}\text { Operative } \\
\text { technique: } \\
\text { cold steel }\end{array}$ & 1.675 & 21.685 & 44.268 & 0.00067 \\
\hline
\end{tabular}

* Outcomes of airway obstruction or adenotonsillar bleed requiring a return to the operating theatre

The four overnight admission categories - mandatory, desirable, convenient and trivial - were created in an attempt to explain the reason for the patient's post-operative stay, and in turn to help identify common characteristics of patients who may not be suitable for day-case tonsillectomy (Table V).

This study considered primary post-tonsillectomy haemorrhage requiring a return to the operating theatre and airway compromise as the most serious complications post-tonsillectomy. Patients at risk of these should always be indicated for a mandatory overnight stay. In this study, 18 patients ( 0.67 per cent $)$ had these complications.

In 28 patients (1.04 per cent), discharge was safe; however, the extra night stay was desirable because of minor adenotonsillar bleeding, sedation or fever. In 63 cases ( 2.33 per cent), the stay was convenient for the patient given the need for analgesic and antiemetic upgrade. In 3 patients ( 0.11 per cent), the stay was considered trivial because of social issues or unrelated symptoms.

\begin{tabular}{lr}
\multicolumn{2}{c}{ TABLE V } \\
OVERNIGHT ADMISSION CATEGORIES FOR PATIENTS \\
WHO REQUIRED ADDITIONAL INTERVENTION \\
\hline Overnight admission category and indications & \multicolumn{1}{c}{ Patients } \\
$(n(\%))$ \\
\hline Mandatory stay & $18(0.67)$ \\
- Primary tonsil bleed requiring return to & $15(0.56)$ \\
operating theatre & \\
- Airway compromise & $3(0.11)$ \\
Desirable stay & $28(1.04)$ \\
- Primary tonsil bleed treated conservatively & $5(0.19)$ \\
- Primary adenoid bleed treated conservatively & $16(0.59)$ \\
- Sedation or GCS score <15 & $2(0.07)$ \\
- Fever & $2(0.07)$ \\
- Intravenous fluids & $3(0.11)$ \\
Convenient stay & $63(2.33)$ \\
- Excessive pain requiring analgesic upgrade & $51(1.89)$ \\
- Excessive nausea requiring anti-emetic upgrade & $12(0.44)$ \\
Trivial stay: social issues, unrelated symptoms & $3(0.11)$ \\
\hline
\end{tabular}

Total $n=112$ (4.15 per cent). GCS $=$ Glasgow Coma Scale 


\section{Discussion}

\section{Synopsis of key results}

The safety of day-case tonsillectomy has been well documented in the literature. ${ }^{3-5,10}$ During the study period, the University Hospital Geelong's practice was to keep all tonsillectomy patients overnight to ensure potential complications were managed. Individual institutional guidelines have not been based on evidence. This study, by investigating the stays of 2863 tonsillectomy patients from June 1998 to June 2014, attempts to produce the basis for future evidence-based, patient selection criteria for day-case tonsillectomy.

The current study suggests that at least 2489 patients (86.94 per cent) did not require the overnight stay. Of the 2700 patients who were kept overnight as part of the University Hospital Geelong's practice, only 211 (7.81 per cent) had a recognised complication, of which only 112 (4.15 per cent) required an intervention. The complication rates in this study are significantly lower than the standards of complication rates set by international studies such as the UK National Prospective Tonsillectomy Audit (overall reported complication rate of 20.4 per cent). ${ }^{11}$

The post-operative haemorrhages reported in this study included those that occurred in the primary (less than 24 hours post-operatively) and secondary (more than 24 hours post-operatively) periods. The results indicate that this hospital achieves a primary post-tonsillectomy haemorrhage rate of 1.48 per cent, with a return to the operating theatre rate of 0.56 per cent, which is also compatible with many national and international studies. ${ }^{11,15}$

There was no significant difference between the use of cold-steel and diathermy techniques in terms of primary post-tonsillectomy haemorrhage patients requiring a return to the operating theatre in this sample $(p=0.11947)$. Cold-steel tonsillectomy had a higher risk of primary post-tonsillectomy haemorrhage requiring a return to the operating theatre when compared with hot monopolar tonsillectomy in the study (1.0 per cent of cold-steel cases needed to return to the operating theatre for bleeding $v s 0.4$ per cent of hot monopolar cases). However, this finding is underpowered and unreliable given the small number of surgeons currently using cold methods at the University Hospital Geelong.

A pre-operative patient questionnaire was used to establish each patient's American Society of Anesthesia score. Bleeding disorders and coagulopathies, such as Von Willebrand disease, were explicitly identified during the adult pre-operative assessment clinic on the patient questionnaire. The paediatric pre-operative questionnaire enquired about the child's co-morbidities in a free-field text box; however, it did not explicitly ask about coagulopathies such as Von Willebrand disease. This has been modified as a result of this study for future paediatric practice.
Not all children undergoing tonsillectomy for sleepdisordered breathing were assessed with sleep oximetry or polysomnography pre-operatively. The authors recognise that this demographic may be at higher risk of post-operative complications; however, sleep studies were not standard protocol at this institution over the entire 16-year time frame and thus were not performed in all paediatric patients.

The authors believe that this study provides an original way of presenting post-tonsillectomy complications and interventions. The creation of the four overnight admission categories allows observations to be made about characteristics shared by the patients in each group, thus identifying favourable and unfavourable characteristics for patients undergoing day-case tonsillectomy, which could be used to inform future practice. The four categories summarise the post-operative interventions that patients required and their subsequent stay as 'mandatory', 'desirable', 'convenient' or 'trivial'.

Table $\mathrm{V}$ indicates that only 18 patients $(0.67$ per cent) developed airway compromise or primary posttonsillectomy haemorrhage requiring a return to the operating theatre (i.e. complications necessitating a mandatory stay). The authors of this study believe that patients at risk of these complications should be recognised and considered unsuitable for day-case tonsillectomy. In this study, these patients had several overlapping characteristics.

Only 24 of the total 2863 patients ( 0.84 per cent) were aged 2 years or less, with the youngest being 11 months old. Of these, three had an airway complication requiring a mandatory stay, which represents 12.50 per cent in this age category. This was a statistically significant finding, and is in line with other literature suggesting that being two years of age or less is a major risk factor for post-tonsillectomy complications and should always indicate a mandatory overnight stay. ${ }^{10,16-18}$

Neoplasm and quinsy were over-represented in those patients who experienced a major bleed with a return to the operating theatre, and were statistically significantly associated with haemorrhage and airway compromise. Ten per cent (3 out of 29) of all neoplasm and 2.3 per cent ( 3 out of 129) of quinsy cases required a mandatory stay, compared with 0.2 per cent of sleep-disordered breathing and 0.4 per cent of tonsillitis cases.

Previous literature also supports the finding that patients with an American Society of Anesthesia score of more than 2 require an overnight stay. ${ }^{16,17}$

These results are largely in agreement with the UK consensus statement on tonsillectomy in children published in $2009 .{ }^{19}$ Thus, the authors believe that patients undergoing tonsillectomy, who are aged two years or less, with tonsil neoplasm or quinsy as indications for tonsillectomy, or an American Society of Anesthesia score of more than 2 because of co-morbidities including Von Willebrand disease, should not be considered for day-case surgery. When these 'high risk' patients were excluded, the rate of complications requiring 
mandatory stay was 0.19 per cent $(n=5)$ in the current study sample. This suggests that the vast majority of patients can be safely discharged home following their procedure, with only a fraction expected to represent in the first 24 hours with a serious complication.

A single, peri-operative intravenous dose of $10-15 \mathrm{mg} / \mathrm{kg}$ tranexamic acid may also help to reduce the incidence of post-operative haemorrhage, and enhance the implementation of safe day-case tonsillectomy. ${ }^{20}$

\section{Study strengths and limitations}

This study spans a 16-year time frame, which has allowed a high patient yield and makes this Australia's largest tonsillectomy study. The thorough investigation of interventions undertaken in the first 24 hours posttonsillectomy produces reliable data concerning the patient characteristics most likely to require post-operative intervention and those least appropriate for daycase surgery. The study assumes that all data reported in the patient notes are accurate and complete.

The data collected are from all surgeons working in the University Hospital Geelong ENT department, including trainee surgeons; thus, the experience of surgeons included in the study is variable and this may have affected the post-operative complication rates.

The study population is heterogeneous. Results have been combined for adults and children, and for all indications and all types of tonsillectomy, including adenotonsillectomy, to gain an overall appreciation of the post-operative outcome. Results have not been analysed separately according to the use of hot or cold surgical techniques, or indications for tonsillectomy.

Not all children underwent sleep oximetry or polysomnography investigations as part of their pre-operative assessment for tonsillectomy. This is a weakness of the study; however, it reflects actual practice at the institution. The authors believe that applying the exclusion criteria in future paediatric cases will avoid the need for significant intervention for breathing complications in the post-operative period.

As this was a retrospective study, there was no standard general anaesthetic, post-operative analgesic or anti-emetic protocol over the course of the 16-year study. Future practice and prospective studies may benefit from a standardised protocol.

Previous literature has shown that bipolar diathermy is superior to monopolar diathermy with regard to thermal tissue damage and subsequent risk of postoperative bleeding. ${ }^{21,22}$ However, for this study, the increased risk of post-operative morbidity has not been quantified, and analysis combines both techniques within the 'hot tonsillectomy' cases.

\section{Interpretation and clinical applicability}

There are several overlapping characteristics for patients who suffered post-operative airway compromise or who required a return to the operating theatre for primary post-tonsillectomy haemorrhage. On this basis, the authors recommend that all elective tonsillectomy patients are screened for their suitability for daycase surgery. We recommend the following exclusion criteria, and suggest that these necessitate overnight observation: age of two years or less; neoplasm or quinsy as the indications for tonsillectomy; an American Society of Anesthesia score of more than 2; or co-morbidities such as Von Willebrand disease (and other coagulopathies). In addition, to further reduce the likelihood of post-operative haemorrhage, each patient may be given a peri-operative dose of $10-15 \mathrm{mg} / \mathrm{kg}$ tranexamic acid intravenously. Further studies are required to assess the impact of these selection criteria on the safety of day-case tonsillectomy.

- A retrospective review was conducted of 2863 tonsillectomies over 16 years at a single Australian institution

- Primary bleeds occurred in 40 cases; 21 were managed medically and 15 required a return to theatre

- Quinsy or tonsil neoplasm as tonsillectomy indications had a higher post-tonsillectomy morbidity risk

- There was no difference in post-operative haemorrhage rates between hot and cold tonsillectomy methods $(p>0.05)$

- Eighty-six per cent of patients did not require overnight stay following tonsillectomy

- The study identified characteristics of patients who should not be considered for day-case tonsillectomy

\section{Acknowledgements}

The authors would like to thank Birmingham Sands Cox Society, the ENT team at the University Hospital Geelong, and supervisors Mr Michael Borschmann and Ebrahim Hassin.

\section{References}

1 Evans A, Khan A, Young D, Adamson R. Assessment of secondary haemorrhage rates following adult tonsillectomy - a telephone survey and literature review. Clin Otolaryngol 2003;28: 489-91

2 Australian Institute of Health and Welfare. Australian hospital statistics 2012-13. In: http://www.aihw.gov.au/WorkArea/ DownloadAsset.aspx?id=60129547000 [1 February 2016]

3 Mills N, Anderson B, Barber C, White J, Mahadevan M, Salkeld L et al. Day stay pediatric tonsillectomy--a safe procedure. Int J Pediatr Otorhinolaryngol 2004;68:1367-73

4 Mahadevan M, van der Meer G, Gruber M, Reed P, Jackson C, Brown C et al. The Starship Children's Hospital tonsillectomy: a further 10 years of experience. Laryngoscope 2016;126: E416-20

5 Lalakea L, Marquez-Biggs I, Messner A. Safety of pediatric short-stay tonsillectomy. Anesthesiology 2000;44:63-4

6 National Institute for Health and Care Excellence. Electrosurgery (diathermy and coblation) for tonsillectomy. Interventional 
procedures guidance (IPG150). In: https://www.nice.org.uk/ guidance/ipg150 [31 December 2015]

7 Ahsan F, Rashid H, Eng C, Bennett D, Ah-See K. Is secondary haemorrhage after tonsillectomy in adults an infective condition? Objective measures of infection in a prospective cohort. Clin Otolaryngol 2007;32:24-7

8 Macfarlane P, Nasser S, Coman W, Kiss G, Harris P, Carney AS. Tonsillectomy in Australia: an audit of surgical technique and postoperative care. Otolaryngol Head Neck Surg 2008;139: 109-14

9 Awori N, King. Chapter 2: Postoperative bleeding, reactionary and secondary haemorrhage. In: Primary Surgery. Oxford: Oxford University Press, 1987:79

10 Pereira H, Xará D, Mendonça J, Santos A, Abelha F. Patients with a high risk for obstructive sleep apnea syndrome: postoperative respiratory complications. Rev Port Pneumol 2013; 19:144-51

11 Royal College of Surgeons England. National Prospective Tonsillectomy Audit Final Report. In: https://www.rcseng.ac. uk/library-and-publications / college-publications / docs / tonsillectomy-audit/ [31 December 2015]

12 Seshamani M, Vogtmann E, Gatwood J, Gibson T, Scanlon D. Prevalence of complications from adult tonsillectomy and impact on health care expenditures. Otolaryngol Head Neck Surg 2014;150:574-81

13 Dhiwakar M, Clement M, Supriya M, McKerrow W. Antibiotics to reduce post-tonsillectomy morbidity (Cochrane Review). Clin Otolaryngol 2010;35:415-16

14 Powell S, Wilson J. Paediatric tonsillectomy - what has the literature added in the last 5 years? The Otorhinolaryngologist 2015;5:139-46

15 Söderman A, Odhagen E, Ericsson E, Hemlin C, Hultcrantz E, Sunnergren $\mathrm{O}$ et al. Post-tonsillectomy haemorrhage rates are related to technique for dissection and for haemostasis. An analysis of 15734 patients in the National Tonsil Surgery Register in Sweden. Clin Otolaryngol 2015;40:248-54
16 Randall D, Hoffer M. Complications of tonsillectomy and adenoidectomy. Otolaryngol Head Neck Surg 1998;118:61-8

17 Biavati M, Manning S, Phillips D. Predictive factors for respiratory complications after tonsillectomy and adenoidectomy in children. Arch Otolaryngol Head Neck Surg 1997;123:517-21

18 Werle A, Nicklaus P, Kirse D, Bruegger D. A retrospective study of tonsillectomy in the under 2-year-old child: indications, perioperative management, and complications. Int $J$ Pediatr Otorhinolaryngol 2003;67:453-60

19 Robb PJ, Bew S, Kubba H, Murphy N, Primhak R, Rollin AM et al. Tonsillectomy and adenoidectomy in children with sleeprelated breathing disorders: consensus statement of a UK multidisciplinary working party. Ann R Coll Surg Engl 2009;91:371-3

20 Robb PJ, Thorning G. Perioperative tranexamic acid in day-case paediatric tonsillectomy. Ann R Coll Surg Engl 2014;96:127-9

21 Sutton PA, Awad S, Perkins AC, Lobo DN. Comparison of lateral thermal spread using monopolar and bipolar diathermy, the Harmonic Scalpel and the Ligasure. Br J Surg 2010;97: 428-33

22 Alkatout I, Schollmeyer T, Nusrat A, Hawaldar MS, Sharma N, Mettler L. Principles and safety measures of electrosurgery in laparoscopy. JSLS 2012;16:130-9

Address for correspondence:

Mr Simon Morris,

3 Orchard Grove,

Morganstown,

Cardiff CF15 8LS,

Wales, UK

E-mail: simonrhysmorris@yahoo.co.uk

Mr S Morris takes responsibility for the integrity of the content of the paper

Competing interests: None declared 